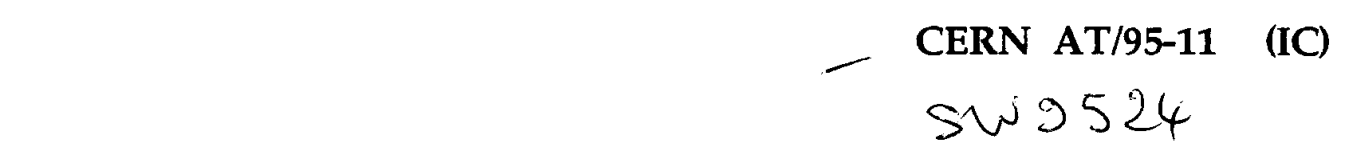

Integrating Industrial and Accelerator Control Systems

R. Saban

CERN LIBRARIES, GENEVA

CERN-AT-95-11

Invited paper to the 1995 Particle Accelerator Conference

1-5 May 1995, Dallas, Texas. 


\title{
INTEGRATING INDUSTRIAL AND ACCELERATOR CONTROL SYSTEMS
}

\author{
R.Saban, AT Division, CERN, 1211 Geneva 23, Switzerland
}

\begin{abstract}
The increasing presence of industrial control systems in high-energy physics laboratories raises the questions of their integration into the general control infrastructure and the organisation of the operation of the associated equipment. The method of integration is dictated by the adopted operation strategy; in turn, the latter determines the former. After an introduction to industrial control systems this paper describes different communication mechanisms with alien systems provided by manufacturers and reports on experience acquired at CERN. The major operation strategies in terms of level of coupling are described and their requirements on the integration methods are discussed.
\end{abstract}

\section{INTRODUCTION}

Commercial industrial control systems have entered high energy laboratory sites either as integral parts of large installations or as embedded controllers hidden in equipment which are largely used in industry $[1,2]$. While it was immediately obvious that the former (cryogenics, tunnel cooling and ventilation, etc.) needed to be monitored and partly controlled because of their tight coupling to accelerator operations, it took some time before it was felt there was a need for integrating the latter (water distribution, building heating, etc.). Furthermore, the picture was blurred by the fact that until recently, some of the installations and equipment were purchased without their control system and were equipped in-house with systems derived from accelerator control architectures. The general belief is that this practice should be abandoned in favour of turnkey systems; therefore the integration issue for all control systems is posed with an increased need of completeness.

\section{CONTROLS IN INDUSTRY}

Industrial control systems were created to automatize manufacturing industry. Broadly speaking, manufacturing processes are of two types: sequential processes (e.g. vehicle assembly) and continuous processes (e.g. oil cracking). This classification has given birth to two lineages in industrial control equipment both best adapted to the type of process to which they are applied: relay based logic gave birth to one and analogue regulators gave birth to the other. Because of the complexity and also the size of the plants, the most advanced techniques for supervision, communication, integration of alien equipment, remote control, etc. were developed by manufacturers of control systems for continuous processes.
Control equipment in industry is based on rugged networks, fieldbuses and digital computers equipped with input/output ports with or without dedicated processing power. They are designed to sustain the harsh factory or outdoor environment. Of their origin they have retained only the programming method: for sequential processes it is largely inspired from drawing relay based circuits (ladder logic) or representing finite state automata (sequential function chart) while for continuous processes it is based on blocks connected to each other by lines (function block).

Standardization efforts $[3,4]$ in the field of industrial controls have led to a widely accepted model known as the Computer Integrated Manufacturing (CIM) pyramid. This model divides the industrial control of a factory into four levels with distinct functions and capabilities.

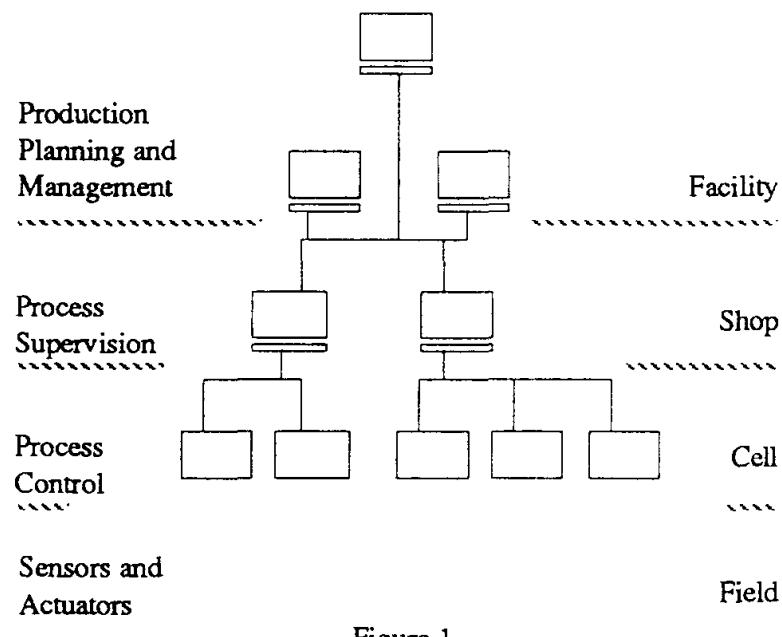

Figure 1

Manufacturers of control systems for industry offer a complete line of products which ranges from the programmable logic controllers (PLC) on the factory floor to the process supervision and advanced production management stations. Additionally, they provide communication mechanisms, supervision tools, archiving schemes, etc. Care must be taken to preserve this bundle of features when integrating an industrial control system, or part of it, in an accelerator control system.

Integration has always been a market penetration asset: in fact, all manufacturers were confronted with existing alien control equipment which needed to be connected to the new system which they were providing. A variety of solutions have been developed.

A. Integrating Industrial Subsystems

Because of their large use in the manufacturing industry, a number of important domains in controls are particularly well understood. These domains include for example motion 
control, control mechanisms for feedback loops and others. Such systems are widely used in accelerator/collider control but despite the existence of a big commercial offer, they are almost always built around in-house developments. However, there are cases where such systems have been integrated into the accelerator control system.

The most common solution connects PLCs at the cell level via a proprietary protocol over RS-232C (see figure 2). While being simple and based on well-known technology, this approach provides little bandwidth and tends to overload the PLC. The latter, which is foreseen for process control, is tied up with tasks like protocol conversion and communication management.

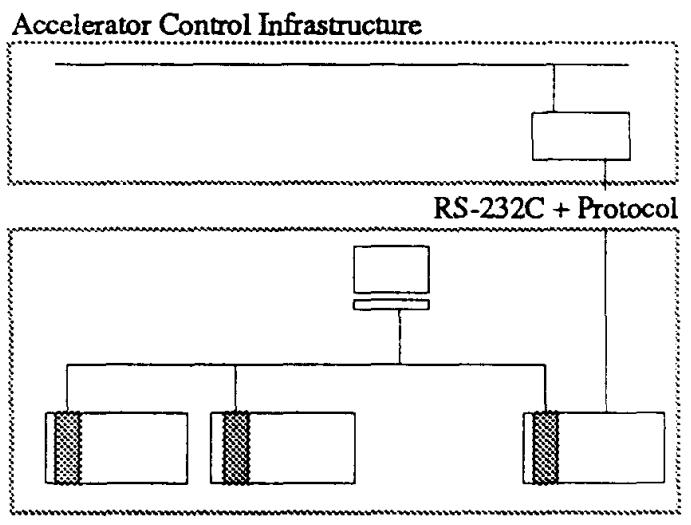

PLC Cluster or Subsystem

W Proprietary Communication System

Figure 2

A variant of this is the integration of a cluster of PLCs acting as an industrial subsystem. This integration can be achieved either using the previous mechanism or exploiting the few other interfacing mechanism foreseen by the manufacturer: these include the porting of an industrial fieldbus to standard computer platforms.

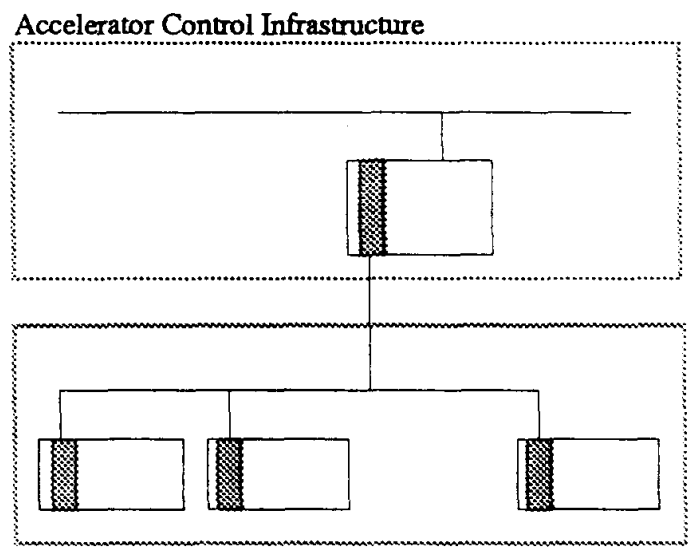

PLC Cluster

Proprietary Communication System

Figure 3
Another variant which has been implemented at CERN consists of using a commercial supervision package [5] which on one hand mediates the interaction with the PLC and on the other hand hosts a programming interface which disguises it in a member of the host control system.

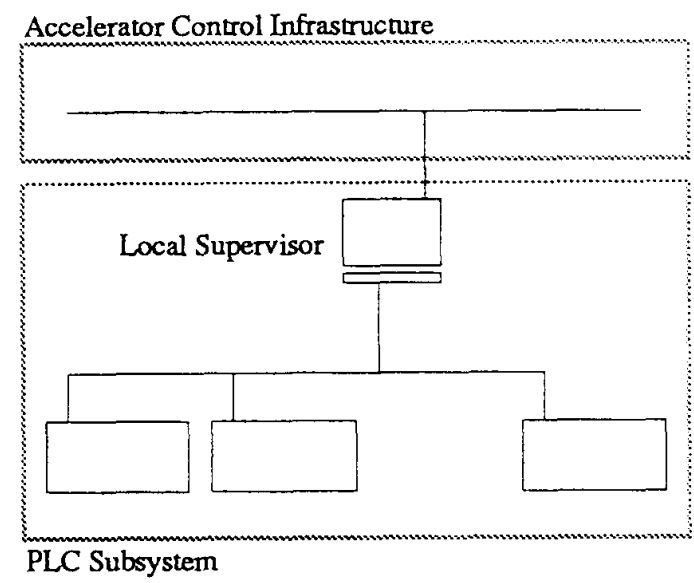

Figure 4

The major advantage of the latter types of integration is that the architecture of the host accelerator system is not altered by the presence of the alien system. The man-machine interface, alarm handling and logging, the communication mechanisms of the host system are used to control and monitor the industrial process below. However, all these facilities already foreseen by the manufacturer tend to be lost in this configuration.

\section{B. Intra-PLC Communications}

Communicating between PLCs of different brands has been made possible by the emergence of the Manufacturing Automation Protocol (MAP) standard $[4,6]$. This protocol has adopted ISO standards for all seven layers of the OSI model. At the highest level, called the Manufacturing Message Specification (MMS), an industrial automation controller is defined as a Virtual Manufacturing Device (VMD) with a number of properties and capabilities: upon the establishment of the service, a system which wants to use the associated equipment interrogates the controller about the particular type, the capabilities, the services, the power of the VMD and uses it accordingly.

A number of classes of devices have been described in companion standards to MAP; they range from Robot Control, Numerical Machines, Production Management to PLCs. The particular services offered by the latter companion standards include program invocation, variable exchange and executable image downloads. All these services are invoked by name and are independent of structures internal to the device or particular to a certain manufacturer.

In time critical applications this protocol is considered too heavy for intra-PLC communications. Therefore, all the PLC manufacturers that have developed proprietary communications mechanisms based on MAP never completely followed the MAP standard for communicating with their own PLCs: rather, they developed two different communication mechanisms running on the same hardware: one to be used with PLCs which they manufacture, the other with alien devices. The first implements a lighter, and 
therefore more performant, version of MAP, while the second follows strictly the MAP standard [7,8]. Since with proprietary communications mechanisms care has been taken to follow the MAP standard at the uppermost layer compatibility, at the application program level, has usually been preserved.

\section{Facility Level Integration}

Manufacturers of large distributed control systems usually supply the process controllers, the supervision consoles and as well the communications infrastructure. The architecture usually includes a gateway to the control system situated in a production/facility management console which is often implemented using a general purpose computer platform. An application program library or a network server gives access to the parameters of the process which usually uses a local real-time database as a stepping stone between the requester of information and the field.

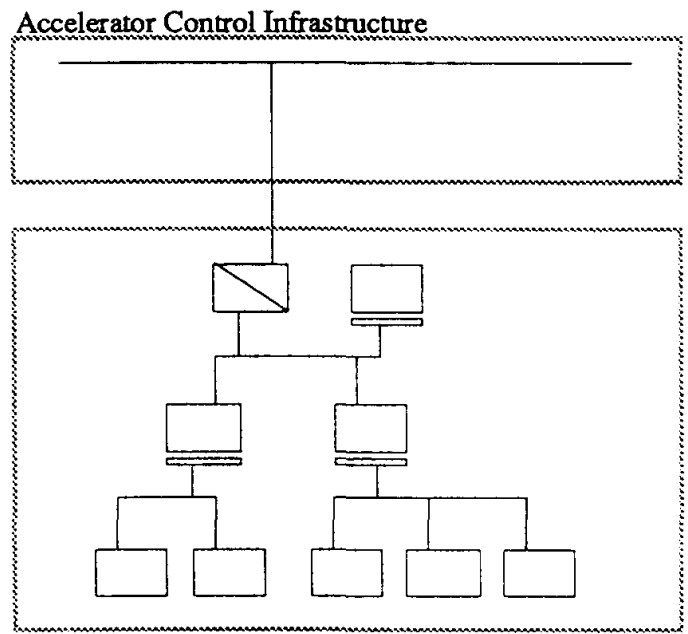

Distributed Control System

Figure 5

\section{ORGANIZATION OF THE OPERATION OF FACILITIES}

A number of large installations, thereafter referenced as facilities, have been integrated into the equipment of the large accelerator/collider installations of today. Not originating from high-energy physics, they provide essential services for the operation of the laboratory. The technology behind them is production oriented and was first developed or has been derived from conventional industrial processes of the same or smaller size. These facilities include air cooling, helium cryogenics, vacuum, ventilation, water distribution, etc. Not recognizing the common feature of their origin, different control strategies have been adopted in the past at CERN: some, like vacuum have completely followed the accelerator control strategy; others, like LEP ventilation and electricity distribution, have followed a mixed strategy and others, like LEP cryogenics, have followed a completely industrial strategy. With the exception of those facilities which are completely industrially controlled and therefore have their own control center, all have developed to a certain extent cryptic activity centers manned by experts. Outside normal working hours, a first line service is provided by operators manning a Technical Control Room.

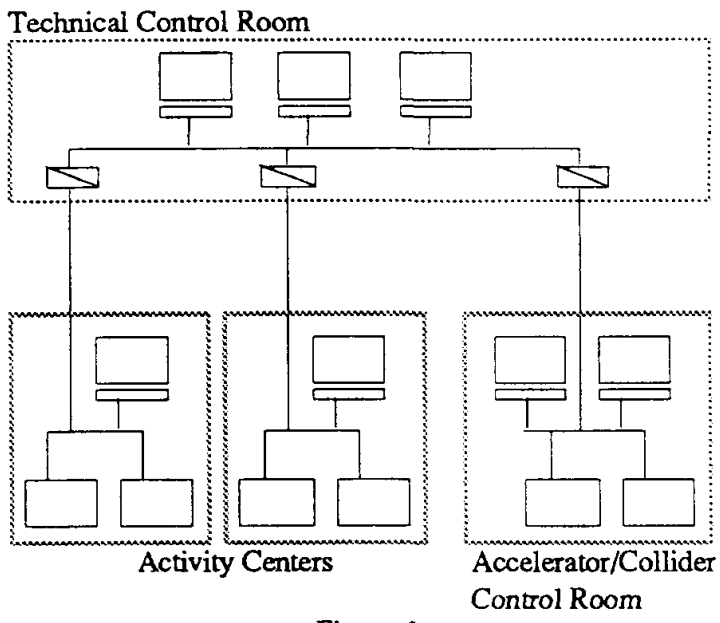

Figure 6

In case of equipment fault, the staff in the Technical Control Room follows a predefined procedure in order to reestablish normal operating conditions. If this fails, the facility is put, or falls by itself, into a safe stand-by state and an equipment specialist is called in to recover the facility. Indeed their complexity makes it hard to employ a team of general purpose operators: rather, in order to diagnose and recover from second level equipment faults, a team of experts in the particular trade is needed. This need becomes absolutely essential for equipment tuning and performance optimization.

In the collider/accelerator control room, the engineer in charge and the operators can do little with information on the speed of a turbine, the temperature in a helium vessel or the pressure in an air duct; rather, they need limited information on the facility as a whole, for example the answer to the question on whether or not they have enough cryogenic power to start an accelerating ramp.

The equipment specialist acts from an activity center; there he finds the tools which allow him to observe the system in depth and detail. He uses the tools which were made available and used during the commissioning of the facility, and are commonly in use by specialists in the same trade elsewhere; therefore, are most mature and suited for regular operation.

In order to achieve this operation strategy three major issues need to be resolved. First, a gateway needs to be programmed/configured in order to provide an access to the industrial system. This must be coherent with the accelerator control system architecture [9].

The second issue is related to network protocols. They should be able to traverse routers and bridges installed on the accelerator control system communication infrastructure and therefore be known by these devices.

The last, but not least important, issue is related to the way the industrial communication mechanisms load the network. In fact, some tend to consider the network as a private communication medium and use the full bandwidth. If this is the case, the demanding (real-time and volume) communications should be conveyed on a dedicated network (figure 7). The three issues have been adressed at CERN; 
some of them have been solved by implementing particular solution; the protocol related issue is currently being investigated [10].

\section{EXPERIENCE AT CERN}

\section{A. Description}

The cryogenics for the LEP2 project follow the facility 'evel integration strategy. A turn-key distributed control system has been specified, purchased and installed in LEP. The CERN communication infrastructure has been used to connect remote sites.

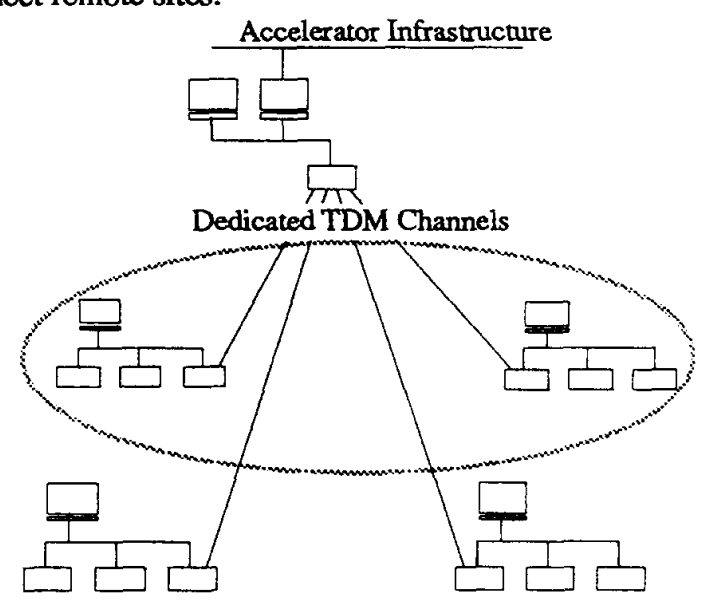

Figure 7

Regional control of the cryogenic installations is done from operator consoles situated at the four even points of LEP. The same control can be exercised remotely from the activity center. A production management system is situated in the activity center and is loosely connected to the accelerator control system.

The cooling and ventilation system of LEP was designed and manufactured by industry and controlled using industrial control systems. They are integrated as an industrial subsystem in the accelerator control system (Figure 2). While local control is possible in the four even points using industrial supervision consoles, the overall system is controlled and monitored via the accelerator control system. In this respect, the industrial subsystem is not different from any other subsystem of the accelerator. The overall cost for such a control system is around $200 \mathrm{SFr}^{1}$ per channel; this does not include the cost of in-house developments, but all the hardware and the software embedded in the PLCs.

Similarly, the control system of the vacuum for ISOLDE is implemented with a set of PLCs connected via a proprietary network interfaced to a front-end IBM PC compatible [11]. The latter performs the function of a gateway to the vacuum system and the protocol conversions. The recently commissioned access control system of the SPS, is in itself a complete industrial system. It has circumvented the integration problem by providing the supervision via operator consoles in the accelerator control room. The latter, for this

11 US $\$ \equiv 1.3 \mathrm{SFr}$, Swiss Banking Corporation, December 1994 particular case, coincides with the activity center. The overall cost for such a control system is around 160 SFr per channel. It includes the hardware, the embedded and supervision software but does not include the cabling of the point-to-point links.

\section{B. Discussion}

From the integration exercises which were successfully conducted at CERN, a few important lessons can be learnt.

In order to keep under control the amount of the work associated to integrating industrial control systems, the number of manufacturers and therefore the number of different systems on the site can be minimized. The majority of the systems, both in volume and number, present on the CERN site are supplied by three major manufacturers. Although natural, this limit can be confirmed by stating the principle in the technical specifications. CERN is better armed for integrating these systems since in-house competence has been developped. The creation of a user group has encouraged people to choose large manufacturers already known at CERN thus contributing to this limitation.

The facility integration strategy which was adopted for the LEP2 cryogenics (figure 7), allowed the specification, purchase and installation of a turn-key commercial industrial control system [12]. This resulted in the full utilisation of all the features commonly used by people in the same trade elsewhere in industry. The major benefit of this was the large bundle of features purchased off-the-shelf and the relative maturity of the system purchased. The loose integration of this system into the accelerator control system was a non-issue because of the choice -by the manufacturer- of a commercial platform (VAX and later HP9000) to gateway its system. A program running under UNIX ${ }^{\mathrm{TM}}$ on a Hewlett-Packard HP9000 computer provides either a regularly refreshed data file or behaves as a data server connected via remote procedure calls or database query languages. This gateway is a delicate point which must be carefully dimensioned and used for what it was designed for: namely, giving access to process variables needed for accelerator operation.

The following two points should be emphasized:

1 For CERN, the tendering of the control system separately from the equipment has been a source of, often intractable, problems in understanding the equipment manufacturers and conveying the information to the control system suppliers. A different approach, namely the purchase of the equipment with its control system should be considered in the future.

2 Existing communication facilities (dedicated Time Division Multiplex channels, Ethernet, etc.) were made available to the control system supplier by CERN. A delicate issue of limits of responsibilities may arise in the future in case of equipment failure.

It is beyond doubt that this approach can only be adopted when the systems concerned are large and the equipment controlled is expensive: the overall cost for such a control system is around $700 \mathrm{SFr}$ per channel. This includes the hardware and the software, from the field to the supervision console as well as the installation and the commissioning of the system.

The industrial subsystem integration strategy which was adopted for the LEP cooling and ventilation system (figures 2 and 4) allowed CERN to benefit partly of the experience of 
equipment and industrial control system manufacturer: in fact, only the process control part was completely dealt with by industrial PLCs. This put the suppliers into a familiar development environment. However, for monitoring and control, the communication mechanisms adopted were those of the host accelerator control system: this resulted in a total loss of the off-the-shelf features normally purchased with a commercial product: namely, intra-PLC communication, supervision, alarm handling, trends, etc. Some of these, absolutely necessary for the operation of the facility, had to be redesigned and, often painfully, integrated into the accelerator control system at a non negligible effort and cost. It is worthwhile mentioning that this approach, for such a big facility, results in an unnecessary tight coupling between the two control systems and the people who developped the interface. This mode of integration was not adapted to the size of the system.

\section{CONCLUSION}

The present technologies used by industrial control system manufacturers permit the easy integration of such systems at the highest level (figures 1 and 5). For large facilities this mode of integration is the most appropriate one because of technical advantages, better cost control and maturity of the products. The operation of such facilities by external teams provided via industrial support contract becomes feasible because of similarities with other industrial installations. In order to ease the integration of facilities following this strategy, the accelerator control system architectures must foresee alien systems and not alien subsystems only. Similarly, at an early stage of the project, the offer of the manufacturer must take into account the integration mechanisms available. The lack of standards probably implies that every system will require its own interface.

The use of industrial subsystems handling tasks widely present in industry like motion control, regulations, sequencing must be encouraged and in-house developments avoided. The inciusion of a limited number of interfaces like MAP and manufacturer dependent MMS implementations in the accelerator control system architectures will permit the natural integration of industrial subsystems.

Because of the size of the accelerator complex, the presence on the site of distributed control systems is unavoidable. The installation of private networks and other communications media have, so far, been avoided whenever this proved possible. In view of the evolution towards an increased number of industrial system and their associated communication mechanisms, it seems reasonable that highenergy physics laboratories foresee the integration of these mechanisms in the palette of network services offered by the laboratory and create a support structure with adequate competence and resources.

\section{ACKNOWLEDGEMENTS}

The present paper is the result of a CERN-wide study that went on in CCIP2 where people recognized the importance

2 Coordination des Contrôles Industriels de Processus, where specialists from different domains regularly meet around and the benefits of adopting commercial solutions and using industrial control systems. I would like to thank in particular P.Ciriani, A.Guiard-Marigny, H-K.Kuhn, P.Liénard and M.Rabany who, with their work or through fruitful discussions, have complemented my knowledge on the subject.

\section{REFERENCES}

[1] M.Rabany, Interfacing Industrial Process Control Systems to LEP/LHC, ICALEPCS, Tsukuba 1991

[2] R.Saban, P.Ciriani, A.Guiard-Marigny, H.Laeger, M.Rabany, A.Swift, Equipment industrially controlled, ICALEPCS, Berlin 1993

[3] International Standard IEC 1131-3, Programmable controllers, 1993

[4] A.Valenzano, C.Demartini, L.Ciminiera, MAP and TOP Communications - Standards and Applications, AddisonWesley 1992.

[5] F.Momal, D.Brahy, R.Saban, P.Sollander, Integrating a commercial industrial control system: a case study, ICALEPCS, Berlin 1993

[6] International Standard ISO/IEC 9506, Industrial Automation Systems - Manufacturing Message Specification, 1990

[7] Siemens, Communication Processor CP143, Users Manual Volume 1 and Volume 2, 1993

[8] Telemecanique, TSX ETH 200 Coupleur OSI Ethernet, User's Manual, 1993

[9] D.Brahy, Une passerelle entre le monde des automates programmables industriels et le contrôle des accélérateurs, Internal Technical Note AT-IC93-05, June 1993. 1995

[10] D.Brahy, P.Liénard, Private Communication, March

[11] D.Brahy, Mise en ceuvre de réseaux locaux industriels SINEC au CERN, CERN Internal Technical Note, ATIC95,April 995

[12] H.K. Kuhn, A.C.Juillerat, M.Rabany, J.C.Wolles, Specification, installation and commissioning of a large industrial constrol system for the LEP2 cryogenics, ICALEPCS, Berlin 1993

topics related to industrial control systems and their manufacturers. 
